\title{
Sincronização de Sistemas a Eventos Discretos com Compartilhamento de Recursos
}

\author{
Lucas A. Floriano* Marcos V. S. Alves* Lilian K. Carvalho* \\ Gustavo S. Viana* João C. Basilio* \\ * Universidade Federal do Rio de Janeiro \\ COPPE - Programa de Engenharia Elétrica \\ 21949-900, Rio de Janeiro, R.J., Brasil, (e-mail: lucasfloriano; \\ mvalves; lilian.carvalho; gustavo.viana; basilio@poli.ufrj.br)
}

\begin{abstract}
In this work, a method is proposed to perform the implementation of the synchronization of discrete event systems with resource sharing, by using the common place technique. Two different approaches are presented for synchronization as well as two algorithms to implement the synchronization by using control interpreted petri net (CIPN). The implementation in a didactic mechatronic plant is carried out through programmable logic controllers (PLCs) connected to each other by means of an industrial communication network and programmed in Ladder logic.

Resumo: Neste trabalho, é proposto um método para realizar a implementação da sincronização de sistemas a eventos discretos com compartilhamento de recursos, utilizando a técnica de lugares comuns. Dois tipos de abordagens para a sincronização são apresentados, assim como dois algoritmos para a implementação adaptados para rede de Petri interpretada para controle (RPIC). A implementação em uma planta mecatrônica didática é realizada através de controladores lógicos programáveis (CLPs) conectados em rede e programados em linguagem Ladder.
\end{abstract}

Keywords: Networked discrete-event systems; Petri nets; common places.

Palavras-chaves: Sistemas a eventos discretos em rede; redes de Petri; lugares comuns.

\section{INTRODUÇÃO}

Nas últimas décadas, o mundo tornou-se cada vez mais integrado através das redes de comunicação. Sistemas industriais estão cada vez mais conectados a essas redes por necessidade de integração ou cooperação de dispositivos geralmente posicionados longe um do outro em um sistema distribuído. Em razão disso, novos conceitos surgiram, como o de indústria 4.0 (Gilchrist, 2016), que possui, entre outras características, a interconectividade entre máquinas, dispositivos e sensores. Neste sentido, sistemas formados pela integração de sistemas de computação, redes de comunicação e processos físicos, chamados de sistemas ciber-físicos, têm recebido atenção considerável na literatura (Jirkovsky et al., 2017).

Dentre as formas de representar sistemas ciber-físicos, podemos citar os sistemas a eventos discretos (SEDs). Um dos formalismos adequados para modelar o comportamento de tais sistemas são as redes de Petri, que possuem a capacidade de modelar diversos comportamentos como simultaneidade, sincronização e compartilhamento de recursos (Liu et al., 2017). Além disso, tal formalismo inclui a possibilidade de descrever o comportamento controlado de SEDs. As redes utilizadas para modelagem de controladores são chamadas redes de Petri interpretadas para controle (RPICs), e incluem ações de controle nos lugares e leituras de sensores nas transições como parte de sua estrutura formal (David \& Alla, 2005).

O projeto do controlador de SEDs é geralmente realizado de duas formas: $(i)$ modela-se o comportamento nãocontrolado do sistema e, após isso, controladores são sintetizados a partir da teoria de controle supervisório, (Vieira et al., 2017) e; (ii) projeto do controlador a partir do comportamento desejado do sistema, usando por exemplo, RPICs (Moreira \& Basilio, 2014). O uso de modelos como RPICs e sua composição para o controle de uma planta pode resultar em uma explosão do espaço de estados para sistemas complexos que envolvem muitos componentes (Iordache et al., 2008). Uma solução para esse problema é implementar controladores por meio de módulos. Neste sentido, para que o sistema funcione adequadamente em conjunto, é necessário sincronizar todos os comportamentos dos componentes do sistema modelados por redes de Petri. Em um contexto de diagnóstico de falhas, Genc \& Lafortune (2007) utilizam lugares comuns entre as redes de Petri que modelam o sistema para capturar o acoplamento de seus vários componentes.

Neste artigo, é proposto um método para implementação de sincronização de módulos independentes que necessitam de integração para compartilhamento de recursos. Tais módulos são modelados por RPICs, cuja integração é feita agregando informações diretamente em sua estrutura. A comunicação entre os módulos é representada por lugares 
comuns (Genc \& Lafortune, 2007). Dois tipos diferentes de abordagens para a sincronização serão apresentados, assim como dois algoritmos para sua implementação. Por fim, o método é implementado em um sistema real por meio de CLPs programados em linguagem Ladder, cuja programação é obtida por meio da conversão de RPIC para Ladder desenvolvida em (Moreira \& Basilio, 2014).

A sincronização de SEDs foi abordada em Viana et al. (2013); Pocci et al. (2014); Zgorzelski \& Lunze (2016); Alves \& Pena (2018). Em nenhum destes trabalhos foi abordado o problema da implementação de um método para sistemas modelados por RPICs cuja sincronização seja feita agregando informações diretamente na estrutura das RPICs. Em Pocci et al. (2014); Alves \& Pena (2018), o problema de sincronização foi tratado no sentido de lidar com recuperação de erros, utilizando palavras de sincronização. Em Zgorzelski \& Lunze (2016), os autores propõem um método para sincronização de sistemas em rede utilizando autômatos I/O. Entretanto, não está claro como fazer a implementação de tal método. Em Viana et al. (2013), é proposto um método que não modifica as RPICs para realizar sincronização de módulos independentes. Um módulo específico é criado e efetuado diretamente no diagrama Ladder para realizar tal tarefa.

Este artigo é organizado da seguinte forma. Na seção 2, apresenta-se uma breve revisão de redes de Petri interpretadas para controle e lugares comuns. A seção 3 apresenta a formulação do problema de sincronização e métodos para solucioná-lo. A seção 4 ilustra os métodos de implementação de sincronização para um sistema de manufatura didático. As conclusões são apresentadas na seção 5 .

\section{FUNDAMENTOS TEÓRICOS}

Antes de apresentarmos a definição de redes de Petri vamos, inicialmente, apresentar a definição de um grafo de uma rede de Petri.

Definição 1. (Grafo de uma rede de Petri) Um grafo de uma rede de Petri é definido como um grafo bipartido $(P, T$, Pre, Post $)$, em que $P$ representa o conjunto finito de lugares, representados graficamente por um círculo, $T$ é o conjunto finito de transições, representadas graficamente por uma linha, Pre $:(P \times T) \rightarrow \mathbb{N}$ é a função de arcos ordinários que ligam lugares a transições e Post : $(T \times$ $P) \rightarrow \mathbb{N}$ é a função de arcos ordinários que ligam transições a lugares, ambos representados graficamente por uma seta.

Para que uma rede de Petri represente um sistema dinâmico, adicionamos à ela uma marcação, que será representada por um vetor linha $x=\left[x\left(p_{1}\right) x\left(p_{2}\right) \ldots x\left(p_{n}\right)\right]$. Graficamente, esta marcação será representada por um número de fichas $x\left(p_{i}\right) \in \mathbb{N}$ distribuídas nos lugares. Estas fichas possuirão a função de habilitar o disparo de uma transição, que por sua vez mudará a marcação da rede assim como seu estado, criando uma dinâmica. Uma transição é dita habilitada quando:

$$
x\left(p_{i}\right) \geq \operatorname{Pre}\left(p_{i}, t_{j}\right), \forall p_{i} \in I_{p}\left(t_{j}\right),
$$

em que $I_{p}\left(t_{j}\right)=\left\{p_{i} \in P: \operatorname{Pre}\left(p_{i}, t_{j}\right)>0\right\}$.

Podemos, agora, definir formalmente redes de Petri.
Definição 2. (Redes de Petri) Uma rede de Petri é uma quíntupla $N_{p}=\left(P, T\right.$, Pre, Post,$\left.x_{0}\right)$, em que $(P, T$, Pre, Post) é o grafo de uma rede de Petri e $x_{0}$ é a marcação inicial do conjunto de lugares.

É possível estender a definição de rede de Petri acrescentando um novo tipo de arco: o arco inibidor. Arcos inibidores ligam um lugar a uma transição impedindo que a transição seja habilitada enquanto o lugar possuir um número de fichas maior ou igual ao peso do arco. Os arcos inibidores são representados graficamente por um arco com um círculo na ponta. A partir desse conceito, pode-se definir uma rede de Petri marcada e estendida.

Definição 3. (Redes de Petri estendidas) Uma rede de Petri marcada estendida é definida como uma sêxtupla $N_{m e}=\left(P, T\right.$, Pre, Post, $\left.x_{0}, I\right)$, em que P, T, Pre, Post e $x_{0}$ são definidos da mesma forma que na definição 2 e $I:(P \times T) \rightarrow \mathbb{N}$ representa o conjunto de arcos inibidores ligando lugares a transições.

Para que as redes de Petri possam ser usadas para representar sistemas reais compostos por sensores e atuadores, novas adições devem ser feitas. Na nova definição, os lugares serão associados a ações e as transições serão associadas a condições e eventos. Além disso, estas serão divididas em dois grupos $T=T_{D} \dot{U} T_{0}$, em que $T_{D}$ representa as transições temporizadas e $T_{0}$ representa as transições não temporizadas. À estrutura contendo todas estas adições, é dada o nome de rede de Petri interpretada para controle (RPIC).

Definição 4. (RPIC) (David \& Alla, 2005) Uma rede de Petri interpretada para controle é definida como uma sêxtupla $N=\left(P, T\right.$, Pre, Post, $\left.x_{0}, I, C, E, l, D, l_{D}, A_{i}, A_{n}, l_{A}\right)$, em que $\left(P, T\right.$, Pre, Post, $\left.x_{0}, I\right)$ é uma rede de Petri estendida segura, $C$ e $E$ são os conjuntos de condições e eventos externos associados às transições não temporizadas $T_{0}$, $l: T_{0} \rightarrow(E \times C)$ é a função que associa cada transição do conjunto $T_{0}$ a um evento e uma condição, $\mathrm{D}$ é o conjunto de atrasos de disparos associados às transições temporizadas $T_{D}, l_{D}: T_{D} \rightarrow D$ é a função que associa cada atraso de disparo às transições do conjunto $T_{D}, A_{i}$ é o conjunto de ações impulsionais, $A_{n}$ é o conjunto de ações contínuas e $l_{A}: P \rightarrow\left(A_{i} \cup A_{n}\right)$ é a função que associa os lugares a uma ação pertencente a $A_{i} \cup A_{n}$.

Um conceito importante para análise de propriedades das redes de Petri é chamado grafo de alcançabilidade (David \& Alla, 2005). O grafo de alcançabilidade de uma rede de Petri $N_{p}$ é composto de vértices que correspondem às marcações alcançáveis e de arcos correspondentes ao disparo de transições que resultam na passagem de uma marcação para outra. Um outro conceito importante que precisamos definir é o de lugares comuns. Um lugar comum pode ser entendido como um lugar compartilhado entre duas ou mais redes de Petri independentes (Genc \& Lafortune, 2007).

Definição 5. (Lugares comuns) Um conjunto de redes de Petri com lugares comuns é definido por $\mathcal{M}=\left\{\left(N_{z}, \mathcal{P}_{z}\right)\right.$ : $z=1,2, \ldots, M\}$, em que $N_{z}$ é uma RPIC e $\mathcal{P}_{z}=\left\{P_{z, i} \subseteq\right.$ $P_{z}: i=1,2, \ldots, M$ e $\left.i \neq z\right\}$ é um conjunto de subconjuntos de $P_{z}$, em que cada $P_{z, i}$ é o subconjunto de lugares comuns entre os módulos $N_{z}$ e $N_{i}$. 


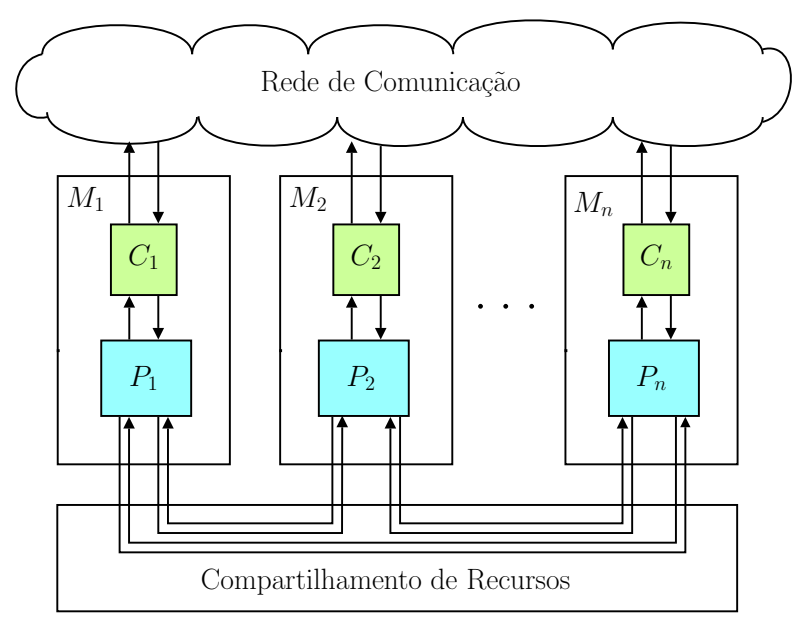

Figura 1. Arquitetura do problema de sincronização.

\section{FORMULAÇÃO DO PROBLEMA}

A arquitetura considerada neste artigo é mostrada na figura 1. O sistema global consiste em um conjunto de módulos $M_{i}$ (subsistemas controlados) tal que controladores $C_{i}$ controlam plantas $P_{i}, i=1,2, \ldots, n$, que interagem entre si compartilhando recursos. Os controladores $C_{i}$ se comunicam por meio de uma rede de comunicação. Consideram-se as seguintes hipóteses.

(1) Não há atrasos nem perdas de pacotes nos canais de comunicação.

(2) Não há um coordenador para o sistema.

Nesta seção, serão apresentados dois métodos de sincronização para a arquitetura apresentada na figura 1 , nos quais os subsistemas controlados são modelados como RPICs acopladas umas às outras através de lugares comuns. Isto é, um controlador $C_{i}$ (módulo $M_{i}$ ) envia uma informação a um outro controlador $C_{j}$ (módulo $M_{j}$ ), que armazena esta informação na própria estrutura da RPIC que modela o módulo $M_{j}$, sincronizando estes dois módulos. A ideia por trás dos algoritmos de implementação desses métodos é modificar as RPICs que modelam cada subsistema controlado, a fim de estabelecer a sincronização dos diferentes módulos que cada RPIC representa.

\subsection{Sincronização por habilitação}

O primeiro método de sincronização será chamado de sincronização por habilitação. Nesta abordagem, queremos que um módulo, denominado escravo, tenha algumas transições $t_{e}$ inicialmente desabilitadas, independentemente da marcação da sua rede de Petri. Apenas quando um outro módulo denominado mestre disparar uma transição $t_{m}$, que as transições $t_{e}$ inicialmente desabilitadas, poderão se tornar habilitadas.

Considere as redes de Petri ilustradas na figura 2. Suponha que o módulo 1 seja o escravo e o módulo 2 seja o mestre, e que as transições $t_{1}$ e $t_{2}$ deverão ser sincronizadas por habilitação. Então dessa forma, mesmo com uma ficha em $p_{1}$, a transição $t_{1}$ deverá estar desabilitada. Apenas quando $t_{2}$ disparar, a transição $t_{1}$ será habilitada. Conforme ilustrado na figura 3(a), isto é possível adicionando a ambas as redes um lugar comum $p_{c}$, de tal forma que $p_{c}$ receba uma

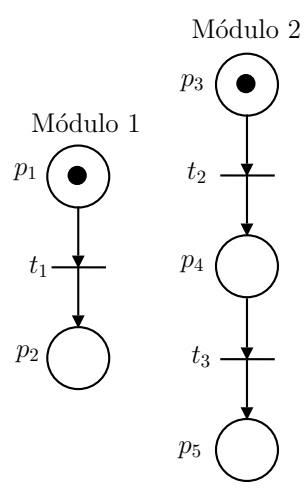

Figura 2. Exemplos de redes de Petri que representam módulos.

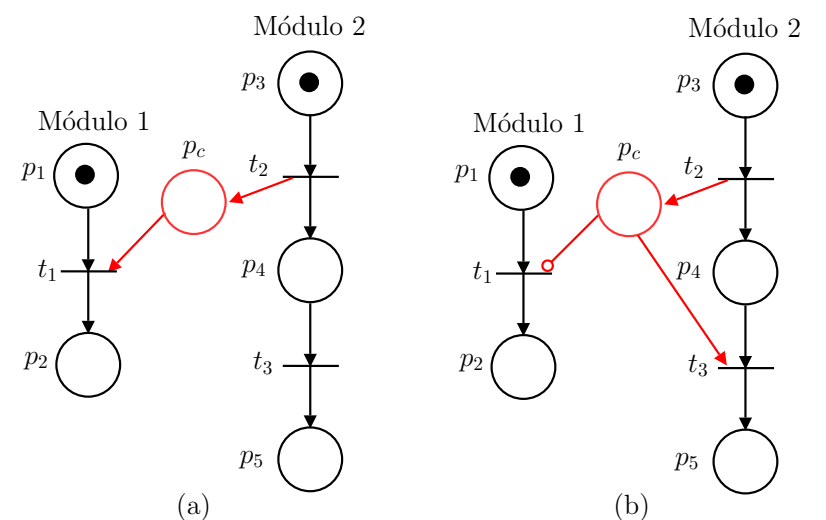

Figura 3. Exemplos de sincronização por habilitação (a) e por bloqueio (b).

ficha somente após o disparo de $t_{2}$ e $t_{1}$ só possa disparar após $p_{c}$ receber uma ficha.

\subsection{Sincronização por bloqueio}

O segundo método de sincronização é chamado de sincronização por bloqueio. Nesse tipo de abordagem, a habilitação das transições $t_{e}$ do módulo escravo (escolhidas para serem sincronizadas) depende, inicialmente, apenas das marcações da sua rede de Petri. Porém, quando o módulo mestre dispara uma determinada transição $t_{m 1}$, as transições $t_{e}$ ficam desabilitadas. Sendo assim, o módulo escravo fica "bloqueado", de tal forma que as transições $t_{e}$ só poderão estar habilitadas novamente após o disparo de uma determinada transição $t_{m 2}$ do módulo mestre.

Considere novamente as redes de Petri ilustradas na figura 2, tal que o módulo 1 é o escravo e o módulo 2 é o mestre. Suponha agora que e que transição $t_{1}$ deva ser sincronizada por bloqueio com $t_{2}$ e $t_{3}$. Dessa forma, $t_{1}$ está inicialmente habilitada porque $p_{1}$ contém uma ficha. Quando $t_{2}$ disparar, a transição $t_{1}$ deverá ficar desabilitada até que $t_{3}$ também seja disparada. Conforme ilustrado na figura 3(b), isto é possível adicionando a ambas as redes um lugar comum $p_{c}$, com um arco inibidor ligando $p_{c}$ a $t_{1}$. Dessa forma, quando $t_{2}$ disparar, $p_{c}$ receberá uma ficha, desabilitando a transição $t_{1}$. Apenas quando $t_{3}$ disparar, o lugar comum $p_{c}$ perderá sua ficha, habilitando a transição $t_{1}$ novamente. 
Vale a pena destacar que, ambos métodos de sincronização definidos nesta seção levam em conta uma ou mais transições do módulo escravo. Já no módulo mestre, a sincronização por bloqueio leva em conta duas transições, enquanto que o sincronização por habilitação, apenas uma.

A escolha pela implementação de um dos métodos de sincronização depende do objetivo do projetista. A sincronização por habilitação é normalmente utilizada quando queremos que o módulo escravo execute uma tarefa $A$ somente depois que o mestre finalizar uma tarefa $B$. Já na sincronização por bloqueio, o módulo escravo não iniciará uma tarefa $A$, enquanto o mestre estiver executando uma tarefa $B$.

\subsection{Alcançabilidade após a sincronização}

Após a definição dos tipos de sincronização, é importante notar que duas condições devem ser respeitadas: $(i)$ a ordem dos disparos de transições dos módulos não deve ser alterada com a sincronização, ou seja, as mesmas marcações ainda devem ser alcançáveis no grafo de alcançabilidade e na mesma ordem, e $(i i)$ os efeitos da sincronização não devem interferir na dinâmica de um módulo mestre, isto é, ele deve "dar ordens" aos escravos, mas não recebêlas. Como consequência, os algoritmos usando lugares comuns para implementar os dois tipos de sincronização devem respeitar as condições $(i)$ e $(i i)$.

Para avaliar os impactos da adição dos lugares comuns, construiremos o grafo de alcançabilidade das redes da figura 2 antes e depois da adição do sincronismo por habilitação, afim de compará-los. As figuras 4(a) e 4(b) ilustram os grafos de alcançabilidade $\mathcal{G}_{1}$ e $\mathcal{G}_{2}$. A composição paralela $\mathcal{G}_{12}$ entre esses grafos é ilustrada na figura 5(a). Note que $\mathcal{G}_{12}$ representa os comportamentos individuais de cada módulo ainda sem sincronização, tal que cada estado do grafo possui a marcação das redes de Petri dos módulos 1 e 2 da figura 2, nesta ordem. Para estabelecer uma comparação, pode-se construir o grafo de alcançabilidade $\mathcal{G}_{c p}$ das redes sincronizadas por habilitação da figura 3(a), considerando que a marcação do lugar comum será a terceira componente do estado do grafo, como ilustrado na figura $5(\mathrm{~b})$.

Note que, de acordo com a figura $5(\mathrm{a})$, é possível alcançar

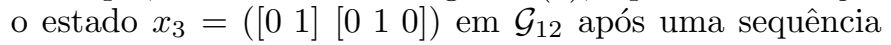
de disparos $t_{1} t_{2}$, isto é, uma sequência em que $t_{1}$ ocorre antes de $t_{2}$. Depois da adição do lugar comum na figura $5(\mathrm{~b})$, este estado só pode ser alcançado após um disparo de $t_{2} t_{1}$, sendo este o objetivo inicial da sincronização. Além disso, comparando a figura 4 e a figura 5(b), é possível verificar que a adição do lugar comum não altera o número de marcações alcançáveis nos módulos e nem a ordem em que são alcançados. Por exemplo, o grafo $\mathcal{G}_{1}$ começa na marcação [1 0 ] e alcança [0 1 l] apenas quando $t_{1}$ é disparada. Isto ocorre também no grafo $\mathcal{G}_{c p}$, ilustrado na figura $5(\mathrm{~b})$. Um outro fato importante é que nenhuma marcação de $\mathcal{G}_{1}$ e $\mathcal{G}_{2}$ deixou de ser alcançada em $\mathcal{G}_{c p}$, sendo que a ordem na qual as marcações aparecem também se manteve.

Uma outra característica importante, que pode ser observada no grafo $\mathcal{G}_{c p}$, é que apenas a transição $t_{2}$ causará uma mudança do estado $x_{0 c p}$ para $x_{1 c p}$, e esta não alterará a

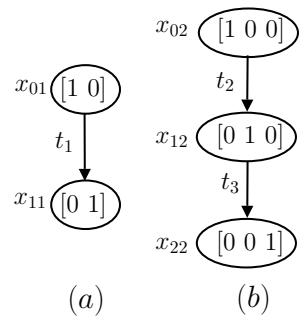

Figura 4. Grafo de alcançabilidade $\mathcal{G}_{1}$ do módulo 1 da figura 2 (a) e grafo de alcançabilidade $\mathcal{G}_{2}$ do módulo 2 da figura 2 (b).

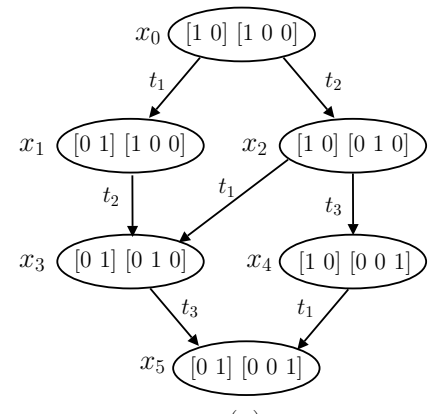

(a)

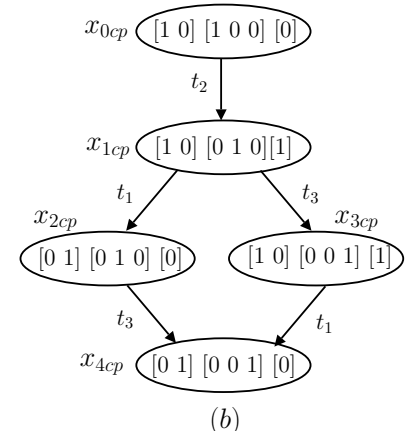

(b)
Figura 5. Composição paralela $\mathcal{G}_{12}$ entre os grafos $\mathcal{G}_{1}$ e $\mathcal{G}_{2}$ da figura $4(\mathrm{a})$ e o grafo $\mathcal{G}_{c p}$ que representa o comportamento sincronizado após a adição do lugar comum (b).

marcação referente ao módulo 1. Isto é, enquanto o sistema se mantiver em $x_{0 c p}$, o módulo 1 não conseguirá mudar de estado, independente de $t_{1}$. Já o módulo 2 não "percebe" os efeitos do sincronismo, visto que o grafo $\mathcal{G}_{c p}$ alcança o estado $x_{3 c p}$ pelo disparo de $t_{2}$ e $t_{3}$ sem que nem mesmo $t_{1}$ tenha disparado. Toda essa análise feita nesta subseção para o método de sincronização por habilitação pode ser realizada para a sincronização por bloqueio da mesma forma.

Uma vez que, a adição de lugares comuns pode realizar a sincronização sem alterar as marcações alcançáveis dos módulos envolvidos, podemos então desenvolver finalmente algoritmos para implementar automaticamente os métodos de sincronização apresentados nesta seção.

\subsection{Algoritmos}

A partir dos dois tipos de sincronismo definidos nesta seção, dois algoritmos podem ser desenvolvidos para implementá-los: o algoritmo 1, para o caso da sincronização por habilitação, e o algoritmo 2 para o caso de sincronização por bloqueio. A ideia por trás dos algoritmos é adicionar os lugares comuns às RPICs de acordo com os métodos definidos na seções 3.1 e 3.2 .

O algoritmo 1 tem como entradas: o número de módulos envolvidos na sincronização $k$ e suas RPICs $N_{j}, g$ grupos $G_{i}$ que formam um par $\left(G_{i}^{\text {mest }}, G_{i}^{e s c}\right)$, em que $G_{i}^{\text {mest }}$ contém a RPIC do módulo mestre e $G_{i}^{e s c}$ as RPICs dos escravos. Mais especificamente, $G_{i}^{m e s t}$ é um par formado pela RPIC do módulo mestre e sua transição a ser sincronizada, enquanto que $G_{i}^{e s c}$ é um conjunto de pares que contêm uma RPIC de módulo escravo e um conjunto de 
transições do respectivo módulo a serem sincronizadas. A saída deste algoritmo será um conjunto de RPICs, modificadas para incluir todos os lugares comuns necessários para a sincronização, assim como seus arcos Pre e Post.

Algoritmo 1. Sincronização de RPICs por habilitação

\section{Entradas:}

- $k$ : número de módulos a serem sincronizados;

- $g$ : número de grupos de sincronização;

- $N_{s}=\left\{N_{1}, \ldots, N_{k}\right\}$, tal que $N_{j}=\left(P_{j}, T_{j}\right.$, Pre $_{j}$, Post $_{j}$, $\left.x_{j}, I_{j}, C_{j}, E_{j}, l_{c j}, D_{j}, l_{D j}, Q_{j}, l_{Q j}\right)$, para $j \in\{1, \ldots, k\}$;

- Grupos: $G_{i}=\left(G_{i}^{\text {mest }}, G_{i}^{e s c}\right), i=1,2, \ldots g$, tal que - $G_{i}^{\text {mest }}=\left(N_{m}, t^{\prime}\right): t^{\prime} \in T_{m}, m \in\{1,2, \ldots, k\}$ $G_{i}^{e s c}=\left\{G_{i, 1}^{e s c}, \ldots, G_{i, p}^{e s c}\right\}$, em que, $\forall w \in\{1, \ldots, p\}$, $G_{i, w}^{e s c}=\left(N_{i, w}, \mathcal{T}_{i, w}\right), N_{i, w} \in N_{s}, N_{i, w} \neq N_{m} \mathrm{e}$ $\mathcal{T}_{i, w} \subseteq T_{i, w}$.

Saída: $N_{s}^{*}=\left\{N_{1}^{*}, \ldots, N_{k}^{*}\right\}$, tal que $N_{j}^{*}=\left(P_{j}^{*}, T_{j}, \operatorname{Pr} e_{j}^{*}\right.$, Post $\left._{j}^{*}, x_{j}, I_{j}, C_{j}, E_{j}, l_{c j}, D_{j}, l_{D j}, Q_{j}, l_{Q j}\right), j \in\{1, \ldots, k\}$.

- Para cada $G_{i}, i=1,2, \ldots g$ :

(1) $L=\emptyset ; P_{i, w}^{*}=P_{i, w} ; P_{m}^{*}=P_{m}$;

(2) Para cada $G_{i, w}^{e s c} \in G_{i}^{e s c}, w=1, \ldots, p$ :

(a) Crie o lugar comum $p_{i w}$;

(b) $L \leftarrow L \cup\left\{p_{i w}\right\}$;

(c) $P_{i, w}^{*} \leftarrow P_{i, w}^{*} \cup\left\{p_{i w}\right\}$;

(d) $\operatorname{Pre}_{i, w}^{*}:\left(P_{i, w}^{*} \times T_{i, w}\right) \rightarrow \mathbb{N}$, tal que:

- $\operatorname{Pr}_{i, w}^{*}\left(p_{i w}, t_{i, w}\right)=1, \forall t_{i, w} \in \mathcal{T}_{i, w}$

- $\operatorname{Pre}_{i, w}^{*}(p, t)=\operatorname{Pre}_{i, w}(p, t), \forall(p, t) \in\left(P_{i, w} \times\right.$ $\left.T_{i, w}\right)$

(3) Para $G_{i}^{m e s t}$ :

(a) $P_{m}^{*} \leftarrow P_{m}^{*} \cup L$

(b) Post $_{m}^{*}:\left(T_{m} \times P_{m}^{*}\right) \rightarrow \mathbb{N}$, tal que:

- Post $_{m}^{*}\left(t^{\prime}, p_{l}\right)=1, \forall p_{l} \in L$

- Post $_{m}^{*}(t, p)=$ Post $_{m}(t, p), \forall(t, p) \in\left(T_{m} \times\right.$ $\left.P_{m}\right)$

Note que no algoritmo 1 , para cada grupo $G_{i}$ de sincronização, nós procedemos da seguinte forma. No passo (1) é definido um conjunto $L$ que receberá todos os lugares comuns criados para aquele grupo. No passo (2), para cada módulo escravo em $G_{i}$, criamos um lugar comum que será adicionado ao conjunto de lugares do módulo escravo já sincronizado, bem como adicionamos um arco partindo de todas as transições daquele módulo envolvidas na sincronização e indo para o lugar comum criado. O processo é repetido para cada escravo, criando um novo lugar comum toda vez. Por fim, no passo (3), todos os lugares comuns criados para dado grupo são adicionados ao conjunto de lugares do mestre daquele grupo, com um arco ligando a transição do módulo mestre escolhida a estes lugares.

No algoritmo 2, teremos as mesmas entradas do algoritmo 1, com a diferença de que o módulo mestre possuirá duas transições envolvidas na sincronização. De forma semelhante ao algoritmo 1, o algoritmo 2 cria um lugar comum para cada grupo e para cada escravo. Desta vez, contudo, o arco conectando os lugares comuns às transições dos escravos será um arco inibidor.
Algoritmo 2. Sincronização de RPICs por bloqueio

\section{Entradas:}

- $k$ : número de módulos a serem sincronizados;

- $g$ : número de grupos de sincronização;

- $N_{s}=\left\{N_{1}, \ldots, N_{k}\right\}$, tal que $N_{j}=\left(P_{j}, T_{j}\right.$, Pre $_{j}$, Post $_{j}$, $\left.x_{j}, I_{j}, C_{j}, E_{j}, l_{c j}, D_{j}, l_{D j}, Q_{j}, l_{Q j}\right)$, para $j \in\{1, \ldots, k\}$;

- Grupos: $G_{i}=\left(G_{i}^{\text {mest }}, G_{i}^{e s c}\right), i=1,2, \ldots g$, tal que - $G_{i}^{m e s t}=\left(N_{m}, t_{m 1}, t_{m 2}\right): t_{m 1}$ e $t_{m 2} \in T_{m}, m \in$ $\{1,2, \ldots, k\}$

$G_{i}^{e s c}=\left\{G_{i, 1}^{e s c}, \ldots, G_{i, p}^{e s c}\right\}$, em que, $\forall w \in\{1, \ldots, p\}$, $G_{i, w}^{e s c}=\left(N_{i, w}, \mathcal{T}_{i, w}\right), N_{i, w} \in N_{s}, N_{i, w} \neq N_{m} \mathrm{e}$ $\mathcal{T}_{i, w} \subseteq T_{i, w}$.

Saída: $N_{s}^{*}=\left\{N_{1}^{*}, \ldots, N_{k}^{*}\right\}$, tal que $N_{j}^{*}=\left(P_{j}^{*}, T_{j}, \operatorname{Pre}_{j}^{*}\right.$, Post $\left._{j}^{*}, x_{j}, I_{j}^{*}, C_{j}, E_{j}, l_{c j}, D_{j}, l_{D j}, Q_{j}, l_{Q j}\right), j \in\{1, \ldots, k\}$.

- Para cada $G_{i}, i=1,2, \ldots g$ :

(1) $L=\emptyset ; P_{i, w}^{*}=P_{i, w} ; P_{m}^{*}=P_{m}$;

(2) Para cada $G_{i, w}^{e s c} \in G_{i}^{e s c}, w=1, \ldots, p$ :

(a) Crie o lugar comum $p_{i w}$;

(b) $L \leftarrow L \cup\left\{p_{i w}\right\}$;

(c) $P_{i, w}^{*} \leftarrow P_{i, w}^{*} \cup\left\{p_{i w}\right\}$;

(d) $I_{i, w}^{*}:\left(P_{i, w}^{*} \times T_{i, w}\right) \rightarrow \mathbb{N}$, tal que:

- $I_{i, w}^{*}\left(p_{i w}, t_{i, w}\right)=1, \forall t_{i, w} \in \mathcal{T}_{i, w}$

- $I_{i, w}^{*}(p, t)=I_{i, w}(p, t), \forall(p, t) \in\left(P_{i, w} \times T_{i, w}\right)$

(3) Para $G_{i}^{\text {mest }}$ :
(a) $P_{m}^{*} \leftarrow P_{m}^{*} \cup L$
(b) Post $_{m}^{*}:\left(T_{m} \times P_{m}^{*}\right) \rightarrow \mathbb{N}$, tal que:
- Post $_{m}^{*}\left(t_{m 1}, p_{l}\right)=1, \forall p_{l} \in L$
- Post $_{m}^{*}(t, p)=$ Post $_{m}(t, p), \forall(t, p) \in\left(T_{m} \times\right.$ $\left.P_{m}\right)$
(c) $\operatorname{Pre}_{m}^{*}:\left(P_{m}^{*} \times T_{m}\right) \rightarrow \mathbb{N}$, tal que:
- $\operatorname{Pre}_{m}^{*}\left(p_{l}, t_{m 2}\right)=1, \forall p_{l} \in L$
- $\operatorname{Pre}_{m}^{*}(p, t)=\operatorname{Pre}_{m}(p, t), \forall(p, t) \in\left(P_{m} \times\right.$

Exemplo 1. Considere as RPICs ilustradas na figura 6 , cujas estruturas de controle foram omitidas, por simplicidade. Suponha que queiramos sincronizar: $(i)$ os módulos 1, 2 e 3 em um grupo, com o módulo 1 como mestre; e (ii) os módulos 3 e 4 em outro grupo, com o módulo 3 como mestre. Para o primeiro grupo, desejamos sincronizar a transição $t_{1}$ do mestre, e as transições $t_{2}, t_{3}$ e $t_{4}$ dos escravos. No segundo grupo, sincronizamos as transições $t_{4}$ e $t_{5}$ dos módulos 3 e 4 , respectivamente.

Definindo as entradas do algoritmo 1 , tem-se que $g=2 \mathrm{e}$ $k=4$. Para os módulos 1, 2, 3 e 4 teremos respectivamente: $N_{1}=\left(P_{1}, T_{1}\right.$, Pre $_{1}$, Post $_{1}, x_{1}, I_{1}, C_{1}, E_{1}, l_{c, 1}, D_{1}, l_{d, 1}, Q_{1}$, $\left.l_{q, 1}\right) ; N_{2}=\left(P_{2}, T_{2}\right.$, Pre $_{2}$, Post $_{2}, x_{2}, I_{2}, C_{2}, E_{2}, l_{c, 2}, D_{2}, l_{d, 2}$, $\left.Q_{2}, l_{q, 2}\right) ; N_{3}=\left(P_{3}, T_{3}\right.$, Pre $_{3}$, Post $_{3}, x_{3}, I_{3}, C_{3}, E_{3}, l_{c, 3}, D_{3}$, $\left.l_{d, 3}, Q_{3}, l_{q, 3}\right) ; N_{4}=\left(P_{4}, T_{4}\right.$, Pre $_{4}$, Post $_{4}, x_{4}, I_{4}, C_{4}, E_{4}, l_{c, 4}$, $\left.D_{4}, l_{d, 4}, Q_{4}, l_{q, 4}\right)$ tais que: $P_{1}=\left\{p_{1}, p_{2}\right\} ; T_{1}=\left\{t_{1}, t_{6}\right\}$; $\operatorname{Pre}_{1}\left(p_{1}, t_{1}\right)=1 ; \operatorname{Pre}_{1}\left(p_{2}, t_{6}\right)=1 ; \operatorname{Post}_{1}\left(t_{1}, p_{2}\right)=$ $1 ;$ Post $_{1}\left(t_{6}, p_{1}\right)=1 ; x_{1}=[10] ; P_{2}=\left\{p_{3}, p_{4}, p_{5}\right\} ; T_{2}=$ $\left\{t_{2}, t_{3}\right\} ;$ Pre $_{2}\left(p_{3}, t_{2}\right)=1$, Pre $_{2}\left(p_{4}, t_{3}\right)=1 ;$ Post $_{2}\left(t_{2}, p_{4}\right)=$ $1, \operatorname{Post}\left(t_{3}, p_{5}\right)=1 ; x_{2}=\left[\begin{array}{ccc}1 & 0 & 0\end{array}\right] ; P_{3}=\left\{p_{6}, p_{7}\right\} ; T_{3}=$ $\left\{t_{4}\right\} ; \operatorname{Pre}_{3}\left(p_{6}, t_{4}\right)=1 ; \operatorname{Post}_{3}\left(t_{4}, p_{7}\right)=1 ; x_{3}=\left[\begin{array}{ll}1 & 0\end{array}\right] ; P_{4}=$ $\left\{p_{8}, p_{9}\right\} ; T_{4}=\left\{t_{5}\right\} ;$ Pre $_{4}\left(p_{8}, t_{5}\right)=1 ;$ Post $_{4}\left(t_{5}, p_{9}\right)=$ $1 ; x_{4}=\left[\begin{array}{ll}1 & 0\end{array}\right]$. Todos os demais elementos de $N_{1}, N_{2}, N_{3}$ e $N_{4}$ que não foram definidos, não são usados pelo algoritmo. 


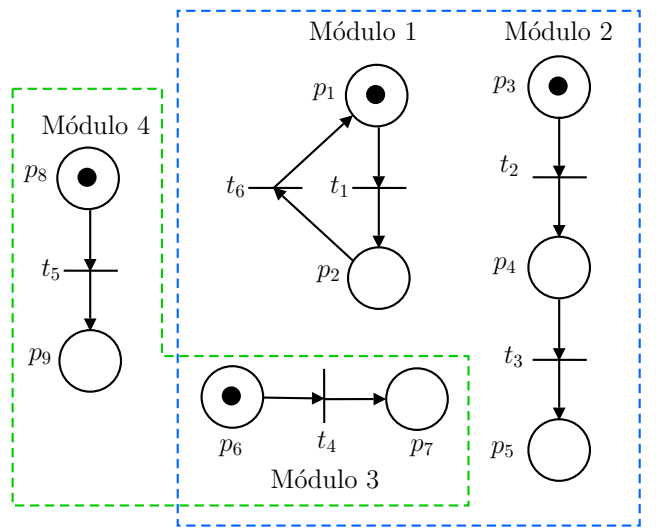

Figura 6. Módulos do exemplo 1.

Note que, o conjunto das RPICs usadas na sincronização é $N_{s}=\left\{N_{1}, N_{2}, N_{3}, N_{4}\right\}$.

Finalmente, os grupos de sincronização são:

$$
\begin{aligned}
& \text { - } G_{1}=\left(G_{1}^{\text {mest }}, G_{1}^{\text {esc }}\right) \text { em que } G_{1}^{\text {mest }}=\left\{\left(N_{1}, t_{1}\right)\right\} \text { e } \\
& G_{1}^{\text {esc }}=\left\{G_{1,1}^{\text {esc }}, G_{1,2}^{\text {esc }}=\left\{\left(N_{2},\left\{t_{2}, t_{3}\right\}\right),\left(N_{3},\left\{t_{4}\right\}\right)\right\}\right. \\
& \text { - } G_{2}=\left(G_{2}^{\text {mest }}, G_{2}^{\text {esc }}\right) \text { em que } G_{2}^{\text {mest }}=\left\{\left(N_{3}, t_{4}\right)\right\} \text { e } \\
& G_{2}^{\text {esc }}=\left\{G_{2,1}^{\text {esc }}\right\}=\left\{\left(N_{4},\left\{t_{5}\right\}\right)\right\} .
\end{aligned}
$$

Aplicando o algoritmo 1 , começando por $G_{1}$, criamos o conjunto $L$ e estabelecemos inicialmente $L=\emptyset$. Sendo assim, selecionamos cada um dos $G_{i, w}^{e s c}$, neste caso, $G_{1,1}^{e s c}$ e $G_{1,2}^{e s c}$. Começando por $G_{1,1}^{e s c}$, seguindo os passos 2.(a), 2.(b) e 2.(c), criamos o lugar comum $p_{11}$ e adicionamos este lugar ao conjunto $P_{2}^{*}$ de $N_{2}^{*}$ e ao conjunto $L$. No passo 2.(d), definimos a função $\operatorname{Pr} e_{2}^{*}$, em que $\operatorname{Pr} e_{2}^{*}\left(p_{11}, t_{2}\right)=1$ e $\operatorname{Pre}_{2}^{*}\left(p_{11}, t_{3}\right)=1$. Note que os pesos de $\operatorname{Pre}_{2}$ são definidos da mesma forma para $\operatorname{Pre}_{2}^{*}$. Voltando para o passo 2 , desta vez com $G_{1,2}^{e s c}$, criamos o lugar comum $p_{12}$ e adicionamos este lugar ao conjunto $P_{3}^{*}$ de $N_{3}^{*}$ e ao conjunto $L$. No passo 2.(d), a função $\operatorname{Pre}_{3}^{*}$ é definida, tal que $\operatorname{Pr}_{3}^{*}\left(p_{12}, t_{4}\right)=1$ em adição aos pesos já definidos em $\operatorname{Pre}_{3}$. Seguindo para o passo 3.(a), formamos o conjunto $P_{1}^{*}$, a partir da união dos conjuntos $L$ e $P_{1}$. No passo 3.(b), definimos a função Post $_{1}^{*}$, em que Post ${ }_{1}^{*}\left(t_{1}, p_{11}\right)=1$ e $\operatorname{Post}_{1}^{*}\left(t_{1}, p_{12}\right)=1$, em adição aos pesos já definidos em Post $_{1}$.

O processo então é repetido, para $G_{2}$, tal que o módulo 3 é mestre e o módulo 4, escravo, da seguinte forma. No passo 1 , criamos novamente o conjunto $L$, inicialmente vazio. Seguindo para o passo 2 , o conjunto $P_{4}^{*}$ é formado pela união do conjunto $P_{4}$ e o lugar comum $p_{21}$. Definimos também a função $\operatorname{Pre}_{4}^{*}$ em que $\operatorname{Pre}_{4}^{*}\left(p_{21}, t_{5}\right)=1$, em adição aos pesos já definidos em $\mathrm{Pre}_{4}$. Finalmente, no passo $3, P_{3}^{*}$ é formado e a função Post $_{3}^{*}$ é definida tal que $\operatorname{Post}_{3}^{*}\left(t_{4}, p_{21}\right)=1$, além de conter os pesos antes definidos em Post $_{3}$.

A sincronização realizada neste exemplo é ilustrada na figura 7, com três lugares comuns criados. Note que, caso a transição $t_{6}$ do módulo 1 não existisse, $t_{3}$ não conseguiria disparar nunca, uma vez que $t_{1}$ precisaria disparar mais uma vez para habilitar $t_{3}$. Este caso mostra que uma escolha ruim de transições para a sincronização pode resultar em um deadlock na rede. Uma análise mais aprofundada sobre as escolhas de sincronismos resultantes em deadlock será feita em trabalhos futuros.

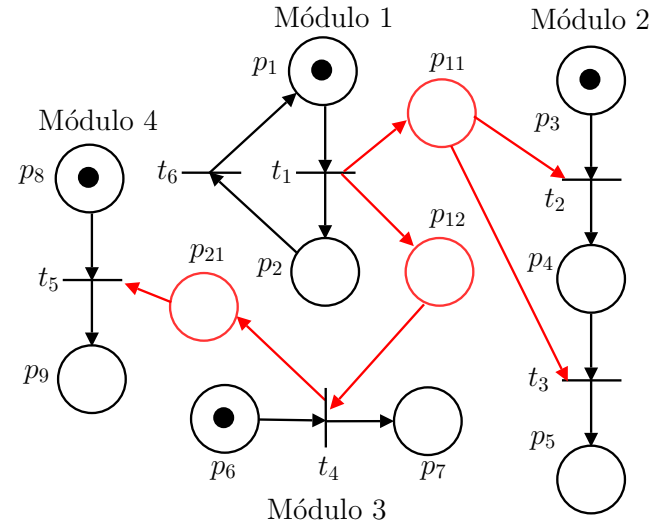

Figura 7. Módulos do exemplo 1 depois da sincronização.

\section{IMPLEMENTAÇÃO EM UM SISTEMA DE MANUFATURA DIDÁTICO}

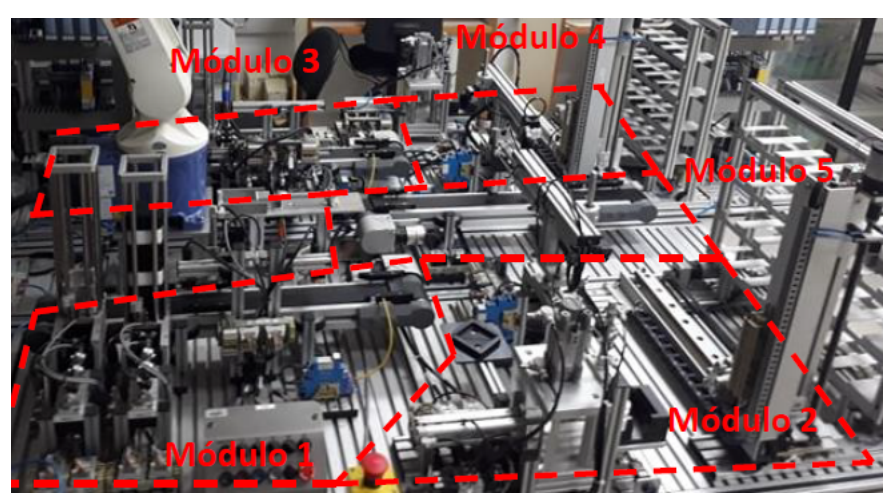

Figura 8. Planta mecatrônica armazenadora de cubos, dividida em módulos.

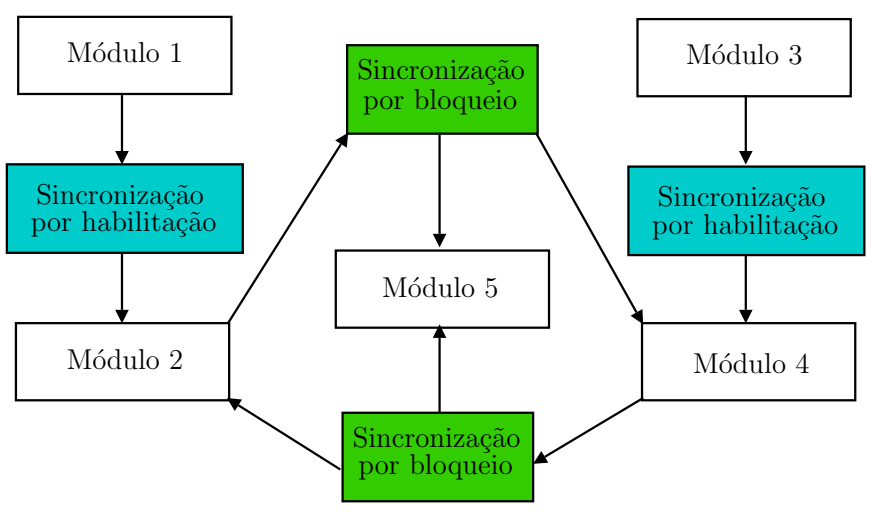

Figura 9. Esquema de sincronização para os módulos da planta mecatrônica ilustrada na figura 8 .

A planta mecatrônica mostrada na figura 8 representa dois sistemas de seleção, montagem e estocagem de peças. $\mathrm{O}$ primeiro sistema é dividido em dois módulos: o módulo 1 realiza a seleção de peças do sistema 1; o módulo 2 recebe as peças selecionadas pelo módulo 1 e pode tanto realizar a montagem e estocagem das peças quanto relocar as peças para o módulo 5 . O módulo 3 realiza a seleção de peças do sistema 2; o módulo 4 recebe as peças selecionadas pelo módulo 3 e pode tanto realizar a montagem e estocagem das peças como relocar as peças para o módulo 5; e o módulo 5 receberá, dos módulos 
2 e 4, peças selecionadas para rejeição e as descartará. Os módulos 2 e 4, contudo, não podem tentar acessar o módulo 5 ao mesmo tempo, uma vez que colisões de seus braços robóticos aconteceriam.

A programação do controle dos módulos é feita em linguagem Ladder, a partir da conversão de RPIC para Ladder desenvolvida por Moreira \& Basilio (2014). Vale a pena comentar que esta programação, assim como as RPICs, são feitas de forma individual e independente, e sincronizações são implementadas para garantir a operação dos módulos em conjunto. Quando um lugar comum recebe uma ficha em uma RPIC de um módulo mestre, essa informação é enviada pela rede de comunicação aos módulos escravos. Como consequência, os lugares comuns de suas RPICs também recebem fichas. A rede de comunicação usada foi implementada com um protocolo baseado no protocolo TCP/IP.

A sincronização entre os módulos da planta mecatrônica armazenadora de cubos, ilustrada na figura 8, é feita da seguinte forma. Primeiramente, queremos que o módulo 2 (resp. 4) seja impedido de iniciar qualquer tarefa que exija uma peça enquanto o módulo 1 (resp. 3) não finalizar seu processo de seleção de peça, disponibilizando a mesma para o módulo 2 (resp. 4). Para um problema com estas características, podemos usar a sincronização por habilitação com o módulo 1 (resp. 3) como mestre e o módulo 2 (resp. 4) como escravo. Com as duas sincronizações por habilitação mencionadas, garantimos que os sistemas 1 e 2 realizem o processo de seleção e montagem de peças adequadamente. Ainda temos, contudo, que garantir o funcionamento adequado do módulo 5 , que é usado para descartes. Como os sistemas 1 e 2 são independentes, é possível que os módulos 2 e 4 tentem acessar o módulo 5 ao mesmo tempo em um dado momento, o que causaria uma colisão entre braços robóticos. Desta forma, queremos que o módulo 4 seja impedido de acessar o módulo 5 enquanto peças vindas do módulo 2 estiverem sendo descartadas, e vice-versa. Note que neste caso, queremos limitar as ações dos módulos apenas em determinados momentos. A sincronização por habilitação não se enquadraria neste cenário, visto que não sabemos qual dos dois módulos, 2 ou 4, tentará acessar o módulo 5 primeiro, e ao aplicar a sincronização por habilitação, uma transição já começaria desabilitada. Sendo assim, a melhor alternativa é usar 2 sincronizações por bloqueio para este caso. A primeira entre os módulos 2 (mestre) e 4 (escravo), bloqueando a transição do módulo 4 que daria início a seu acesso ao módulo 5. A segunda será idêntica à primeira, desta vez com o módulo 4 como mestre e o 2 como escravo. Assim, é garantido que em nenhum momento os dois módulos tentarão acessar o módulo 5 simultaneamente. A figura 9 ilustra o esquema de sincronização entre os módulos da planta mecatrônica armazenadora de cubos. Já o diagrama Ladder e as RPICs referentes à implementação descrita nesta seção podem ser encontrados no link: www.dropbox.com/sh/ gu1xtfdf6z9mcaf/AADvD1ZF_YZ_AadPf7QYEzx_a?dl=0.

\section{CONCLUSÃO}

Neste trabalho, propomos métodos para implementação de sincronização de sistemas a eventos discretos que necessitam de integração para compartilhamento de recursos.
Os subsistemas controlados são modelados por RPICs, cuja integração é representada por lugares comuns. Foram apresentados dois tipos diferentes de abordagens para sincronização, assim como algoritmos para suas implementações. Por fim, os métodos de sincronização propostos foram implementados numa planta mecatrônica didática. Como trabalho futuro, pretendemos aprofundar a análise da alcançabilidade das redes após a sincronização, para identificar e prevenir possíveis bloqueios. Além disso, planejamos estender a abordagem desenvolvida por Moreira \& Basilio (2014) para sistemas conectados em rede, e lidar com a existência de atrasos de comunicação.

\section{REFERÊNCIAS}

Alves, L.V.R. \& Pena, P.N. (2018). Sincronização em sistemas a eventos discretos. Congresso Brasileiro de Automática, XXII CBA, João Pessoa, Brasil.

David, R. \& Alla, H. (2005). Discrete, continuous, and hybrid Petri nets, volume 1. Springer.

Genc, S. \& Lafortune, S. (2007). Distributed diagnosis of place-bordered Petri nets. IEEE Transactions on Automation Science and Engineering, 4(2), 206-219.

Gilchrist, A. (2016). Industry 4.0: the industrial internet of things. Apress, Berkeley, 1st edition.

Iordache, M.V., Antsaklis, P.J., \& Lin, F. (2008). Supervisory control of concurrent systems: A Petri net structural approach. IEEE Transactions on Automatic Control, 53(1), 441.

Jirkovsky, V., Obitko, M., \& Marik, V. (2017). Understanding data heterogeneity in the context of cyber-physical systems integration. IEEE Transactions on Industrial Informatics, 13(2), 660-667.

Liu, G., Zhou, M., \& Jiang, C. (2017). Petri net models and collaborativeness for parallel processes with resource sharing and message passing. ACM Transactions on Embedded Computing Systems (TECS), 16(4), 113.

Moreira, M.V. \& Basilio, J.C. (2014). Bridging the gap between design and implementation of discrete-event controllers. IEEE Transactions on Automation Science and Engineering, 11(1), 48-65.

Pocci, M., Demongodin, I., Giambiasi, N., \& Giua, A. (2014). Testing experiments on synchronized Petri nets. IEEE Transactions on Automation Science and Engineering, 11(1), 125-138.

Viana, G.S., M.V.Moreira, \& J.C.Basilio (2013). Implementação de controladores a eventos discretos usando diagrama ladder com módulo sincronizante. Simpósio Brasileiro de Automação Inteligente, XI SBAI, Fortaleza, Brasil.

Vieira, A.D., Santos, E.A.P., de Queiroz, M.H., Leal, A.B., de Paula Neto, A.D., \& Cury, J.E. (2017). A method for PLC implementation of supervisory control of discrete event systems. IEEE Transactions on Control Systems Technology, 25(1), 175-191.

Zgorzelski, M. \& Lunze, J. (2016). A method for the synchronisation of networked discrete-event systems. In 2016 13th International Workshop on Discrete Event Systems (WODES), 444-451. IEEE. 